\title{
Detecção amperométrica de sulfanilamida sobre eletrodo de carbono vítreo modificado com complexo de cobalto
}

\author{
Maria de Lourdes S. Vasconcellos $(P G)^{1-2 *}$; Edervaldo Buffon $(P G)^{1-3}$; \\ Luciene P.R. Profeti $(\mathrm{PQ})^{1}$; Demetrius Profeti $(\mathrm{PQ})^{1}$ \\ ${ }^{1}$ Departamento de Química e Física, Universidade Federal do Espírito Santo, Alegre, ES, Brasil. \\ ${ }^{2}$ Programa de Pós-Graduação em Química, Universidade Federal do Espírito Santo, Vitória, ES, Brasil \\ ${ }^{3}$ Programa de Pós-Graduação em Química, Departamento de Química Analítica, Instituto de Química de Araraquara, \\ Universidade Estadual Paulista Júlio de Mesquita Filho, Araraquara, SP, Brasil. \\ *e-mail: lourdes-vasconcellos@hotmail.com
}

\begin{abstract}
Resumo
Neste trabalho, um eletrodo de carbono vítreo foi modificado com o complexo hexacianoferrato de cobalto (CoHCF) pela técnica de voltametria cíclica. Este eletrodo modicado apresentou uma considerável estabilidade nas condições empregadas e comportamento eletroquímico influenciado pelos cátions e ânions presentes no eletrólito suporte. Através deste estudo, comprovou-se que as reações redox superficiais apresentam maior reversibilidade na presença do íon potássio, o qual possui maior capacidade de inserção na estrutura do complexo. A análise do comportamento do filme de CoHCF na presença da sulfanilamida foi realizado através da técnica de amperometria. Os resultados obtidos apresentaram uma faixa linear de 8 a $16 \times 10^{-5} \mathrm{~mol} \mathrm{~L}^{-1}$, indicando a possibilidade de aplicação deste eletrodo na detecção da molécula de sulfanilamida, a qual está presente em diversos medicamentos.
\end{abstract}

\begin{abstract}
In this work, a glassy carbon electrode was modified with cobalt hexacyanoferrate complex (CoHCF) by cyclic voltammetry technique. This modified electrode showed a considerable stability at the conditions employed and electrochemical behavior influenced by cations and anions present in the supporting electrolyte. This study showed that the redox reactions in surface of the electrode is highly reversible in the presence of potassium ion, which has a greater insertion capacity in structure of the complex. The analysis of the CoHCF film behavior in the presence of sulfanilamide was performed by amperometry. The results obtained showed a linear range of 8 to $16 \times 10^{-5} \mathrm{~mol} \mathrm{~L}^{-1}$ indicating the possibility of application this electrode in detecting of the sulfanilamide molecule, which is present in several medications.
\end{abstract}

Keywords (Palavras chaves): Eletrodo Modificado, Hexacianoferrato de Cobalto e Sulfanilamida.

\section{Introdução}

A sulfanilamida, composto pertencente à classe das sulfonamidas, é encontrada em medicamentos que atuam no combate de doenças bacterianas, uma vez que ela atua como um inibidor competitivo da enzima bacteriana sintetase de dihidroperoato que catalisa a síntese de ácido fólico, que por sua vez é essencial para a síntese do DNA e RNA bacteriano. O sulfametoxazol, um derivado da sulfanilamida, têm sido largamente empregado no tratamento da Síndrome da Imunodeficiência Adquirida (AIDS), entretanto, a ingestão de concentrações elevadas de sulfonamidas em geral pode causar reações adversas tais como o desenvolvimento da hipersensibilidade do tipo retardado e da hepatite [1]. A ANVISA (Agência Nacional de Vigilância Sanitária) estabelece um limite de ingestão diária de sulfonamidas de até $100 \mu \mathrm{g} \mathrm{kg}^{-1}$ para que não ocorra perigo de intoxicação humana [2]. Diante disso, há um grande interesse de pesquisadores da área da saúde no desenvolvimento de métodos eficazes para a análise da sulfanilamida.

As técnicas eletroanalíticas que utilizam os eletrodos quimicamente modificados (EQMs) tem sido de grande importância para 0 desenvolvimento de diversos estudos, uma vez que a superfície do eletrodo é uma poderosa ferramenta analítica. Este dispositivo quando comparado aos eletrodos convencionais apresentam 
alta sensibilidade e seletividade devido ao maior controle da interface eletrodo/solução proporcionada pelo agente modificador. Este controle adicional impede que fenômenos indesejáveis possam ocorrer durante a realização das eletroanálises, tornando o método mais eficiente para a análise da molécula de interesse [3].

Dentre as espécies modificadoras da superfície de eletrodos, o íon hexacianoferrato destacam-se devido a suas características de formar complexos com uma grande variedade dos metais de transição. Dentro da classe dos hexacianoferratos de metais (MHCF), o hexacianoferrato de cobalto (CoHCF) possui propriedades capazes de interagir com diversas espécies de interesse analítico, bem como apresentar propriedades e atividades eletrocatalíticas significativas [4].

Nesse contexto, o objetivo deste trabalho constituiu em sintetizar um filme de hexacianoferrato de cobalto (CoHCF) sobre a superfície de um eletrodo de carbono vítreo $(\mathrm{CV})$ e verificar sua interação com a molécula de sulfanilamida.

\section{Materiais e Métodos}

O aparato experimental utilizado para a realização deste trabalho consiste numa célula eletroquímica composta por três eletrodos: um fio de platina como contra eletrodo, um $\mathrm{Ag} / \mathrm{AgCl}$ em $\mathrm{KCl}$ saturado usado como referência e um disco de carbono vítreo com 3 $\mathrm{mm}$ de diâmetro e aproximadamente $7,068 \mathrm{~mm}^{2}$ de área usado como eletrodo de trabalho. Para a realização de todos os estudos eletroquímicos utilizouse um Potenciostato/Galvanostato AUTOLAB PGSTAT $128 \mathrm{~N}$ controlado pelo software GPES 4.9.

Os reagentes utilizados neste trabalho foram: $\mathrm{CoCl}_{2}$, $\mathrm{K}_{3}\left[\mathrm{Fe}(\mathrm{CN})_{6}\right], \mathrm{KCl}, \mathrm{K}_{2} \mathrm{SO}_{4}, \mathrm{NaCl} ; \mathrm{NaNO}_{3}$ e $\mathrm{LiCl}$, sendo todos de pureza analítica (PA). Todas as soluções foram preparadas utilizando água ultrapura obtida através do purificador de água MEGAPURITY - MEGA RO/UP.

Antes de qualquer modificação da superfície do eletrodo de CV, o mesmo foi submetido a um polimento mecânico com alumina de granulometria $0,05 \mu \mathrm{m}$, até que sua superfície adquirisse um aspecto espelhado. Após este procedimento, o eletrodo de CV foi imerso em uma solução contendo $9 \mathrm{mmol} \mathrm{L}^{-1}$ de $\mathrm{CoCl}_{2}$ e 3,0 $\mathrm{mmol} \mathrm{L}^{-1}$ de $\mathrm{K}_{3}\left[\mathrm{Fe}(\mathrm{CN})_{6}\right]$ em $0,1 \mathrm{~mol} \mathrm{~L}^{-1}$ de $\mathrm{KCl}$ e submetido a 20 ciclos de varredura de potenciais em um intervalo de 0,0 a $0,8 \mathrm{~V}$ vs. $\mathrm{Ag} / \mathrm{AgCl}$ numa velocidade de varredura de $50 \mathrm{mV} \mathrm{s}^{-1}$ para 0 crescimento do filme de CoHCF sobre a superfície do eletrodo convencional de CV.
Após a preparação do eletrodo modificado, o mesmo foi avaliado quanto a sua estabilidade, a influência da concentração do eletrólito suporte em seu comportamento eletroquímico, bem como seu comportamento eletroquímico na presença de diferentes cátions e ânions. Por fim, foi avaliada a interação entre o filme de CoHCF e a molécula de sulfanilamida aplicando-se uma faixa de potencial adequada para o complexo adsorvido na superfície do eletrodo de $\mathrm{CV}$.

\section{Resultados e Discussão}

A primeira etapa deste trabalho consistiu na eletrossíntese do composto de coordenação hexacianoferrato de cobalto (CoHCF) sobre a superfície do eletrodo de CV utilizando a técnica de voltametria cíclica. A Figura 1 mostra o crescimento do filme de CoHCF no intervalo de potenciais de 0,0 a $0,8 \mathrm{~V}$ durante sucessivos ciclos de varredura a $50 \mathrm{mV} \mathrm{s}^{-1}$ na presença de $9,0 \mathrm{mmol} \mathrm{L}^{-1}$ de $\mathrm{CoCl}_{2}$ e 3,0 $\mathrm{mmol} \mathrm{L}^{-1}$ de $\mathrm{K}_{3}\left[\mathrm{Fe}(\mathrm{CN})_{6}\right]$, utilizando uma solução de $\mathrm{KCl}$ $0,1 \mathrm{~mol} \mathrm{~L}^{-1}$ como eletrólito suporte. Na Figura 1 é possível observar o aumento das correntes dos picos catódicos e anódicos, indicando um processo de eletrodeposição sobre a superfície do eletrodo de CV.

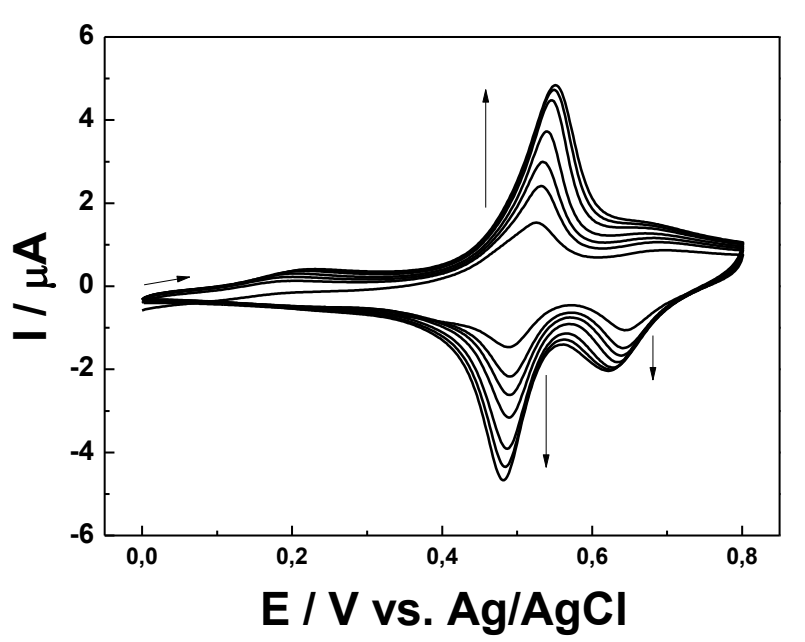

Figura 1: Voltamogramas cíclicos obtidos durante a modificação do eletrodo de CV com CoHCF, através da técnica de voltametria cíclica utilizando uma faixa de potencial de 0,0 a $0,8 \mathrm{~V}$ durante 20 ciclos em velocidade de varredura de $50 \mathrm{mV} \mathrm{s}^{-1}$.

Os picos observados na Figura 1 indicam um comportamento de reversibilidade das reações redox que ocorrem na superfície do filme de CoHCF, as quais ocorrem em aproximadamente 0,48 e $0,55 \mathrm{~V}$ vs. $\mathrm{Ag} / \mathrm{AgCl}$. Estes potenciais de pico podem ser atribuídos aos pares Co"↔ $\mathrm{Co}^{\text {III }}(0,48 \mathrm{~V})$ e $\mathrm{Fe}^{\prime \prime} \leftrightarrow \mathrm{Fe}^{\mathrm{III}}(0,55 \mathrm{~V})$. Tal comportamento também foi observado por Arduini et al. [5] e pode ser descrito pelas seguintes reações respectivamente:

$$
\mathrm{K}_{2} \mathrm{Co}^{\prime \prime}\left[\mathrm{Fe}^{\prime \prime}(\mathrm{CN})_{6}\right] \leftrightarrow \mathrm{KCo}^{\prime \prime \prime}\left[\mathrm{Fe}^{\prime \prime}(\mathrm{CN})_{6}\right]+\mathrm{K}^{+}+\mathrm{e}^{-}
$$




$$
\left.\mathrm{K}_{2} \mathrm{Co}_{3}{ }^{\prime \prime}\left[\mathrm{Fe}{ }^{\prime \prime} \mathrm{CN}\right)_{6}\right] \leftrightarrow \mathrm{Co}_{3}{ }^{\prime \prime \prime}\left[\mathrm{Fe}^{\prime \prime \prime}(\mathrm{CN})_{6}\right]+2 \mathrm{~K}^{+}+2 \mathrm{e}^{-}
$$

Posteriormente, foi realizado um estudo de estabilidade do eletrodo modificado para avaliar a possibilidade de sua utilização em diferentes condições de análise sem a perda da área ativa ou lixiviação do filme de CoHCF. Para esse estudo, o eletrodo foi imerso numa solução de $\mathrm{KCl} 0,1 \mathrm{~mol} \mathrm{~L}^{-1}$ e aplicou-se 250 ciclos sucessivos de varredura de potenciais numa faixa de potencial de 0,0 a $0,8 \mathrm{~V}$, utilizando uma velocidade de varredura de $50 \mathrm{mV} \mathrm{s}^{-1}$, conforme mostrado na Figura 2.

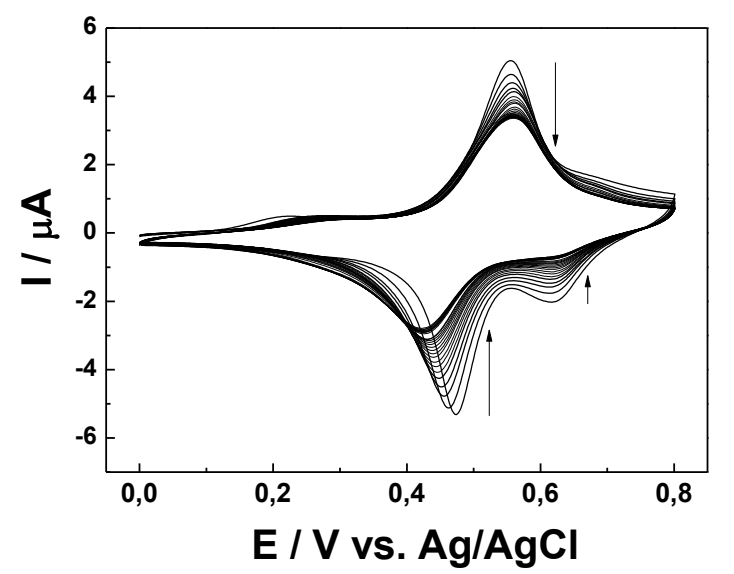

Figura 2: Estabilidade do filme de CoHCF em solução $\mathrm{KCl} 0,1 \mathrm{~mol} \mathrm{~L}^{-1}$ durante 250 ciclos sucessivos numa velocidade de varredura de $50 \mathrm{mV} \mathrm{s}^{-1}$.

Nesta figura observou-se que o perfil voltamétrico apresentou um decréscimo de 33,39\% na intensidade de corrente de pico, fato que pode ser atribuído à dissolução gradual do complexo na solução de eletrólito.

Com o intuito de verificar a influência da natureza do eletrólito suporte no comportamento do filme de CoHCF, o mesmo foi avaliado em diferentes eletrólitos, conforme mostrado na Figura 3.

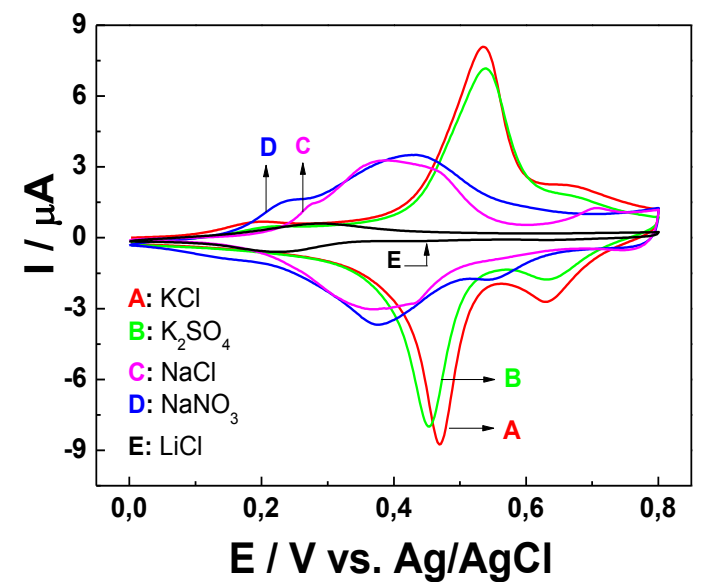

Figura 3: Comportamento ciclovoltamétrico do filme $\mathrm{CoHCF}$ na presença de diferentes eletrólitos: $\mathrm{KCl}, \mathrm{K}_{2} \mathrm{SO}_{4}, \mathrm{NaNO}_{3}, \mathrm{NaCl}$ e $\mathrm{LiCl}$ na concentração de $0,1 \mathrm{~mol} \mathrm{~L}^{-1}$ numa velocidade de varredura de potenciais de $20 \mathrm{mV} \mathrm{s}^{-1}$.
Nesta figura é possível observar o efeito de cátions $\left(\mathrm{K}^{+}\right.$, $\mathrm{Na}^{+}$e $\left.\mathrm{Li}^{+}\right)$e ânions $\left(\mathrm{Cl}^{-}, \mathrm{NO}_{3}^{-}\right.$e $\left.\mathrm{SO}_{4}{ }^{2-}\right)$ no comportamento eletroquímico do complexo. O filme de CoHCF na presença dos eletrólitos $\mathrm{KCl}, \mathrm{NaCl}$ e $\mathrm{LiCl}$ apresentou deslocamento do pico redox para potenciais mais negativos, fato que pode ser explicado pela inserção/de-inserção do cátion de metal alcalino nos canais presentes na estrutura cristalina do complexo durante os processos de transferência de carga. Os picos redox do filme na presença de $\mathrm{K}^{+}$são mais reversíveis e mais definidos, pois os íons potássio participam efetivamente dos processos superficiais de oxidação e redução do filme devido à maior mobilidade iônica e à melhor condutividade em soluções aquosas quando comparado ao $\mathrm{Na}^{+}$e $\mathrm{Li}^{+}$[6-8].

$\mathrm{Na}$ presença dos eletrólitos $\mathrm{KNO}_{3}$ e $\mathrm{K}_{2} \mathrm{SO}_{4}$, o comportamento eletroquímico do filme CoHCF permaneceu o mesmo, exceto pela diminuição na intensidade das correntes de pico, fato que pode ser atribuída pela possível interação entre o filme de CoHCF com o íon sulfato. A mesma diminuição de intensidade de corrente de pico foi verificada nos voltamogramas cíclicos do filme de CoHCF na presença de $\mathrm{NaCl}$ e $\mathrm{NaNO}_{3}$, mostrando possível interação dos cátions e dos ânions destes eletrólitos com o filme de CoHCF.

O complexo de hexacianoferrato de cobalto possui estrutura cristalina cúbica de face centrada com coordenação octaédrica dos íons cobalto e ferro ligados por grupos cianetos [6]. Nesta estrutura, todos os átomos de ferro se coordenam aos átomos de carbono do grupo cianeto, enquanto que todos os átomos de cobalto se coordenam aos átomos de nitrogênio. Átomos de cobalto e de ferro se alternam nos vértices da estrutura cúbica e o centro do retículo cristalino é ocupado por cátions de metais alcalinos de forma a garantir a eletroneutralidade do composto [7]. Desta forma, a presença de íons de metais alcalinos e a força iônica da solução podem influenciar no comportamento eletroquímico do filme de CoHCF, principalmente no que diz respeito a reversibilidade dos processos redox existentes.

Após verificado que o filme de CoHCF apresentou um melhor comportamento eletroquímico na presença do eletrólito suporte de $\mathrm{KCl}$, realizou-se um estudo da variação da concentração deste eletrólito, conforme se observa na Figura 4. Notou-se que o comportamento eletroquímico do filme de CoHCF em soluções de $\mathrm{KCl}$ com diferentes concentrações permanece inalterado, contudo os valores dos potenciais dos picos redox aumentam conforme aumenta a concentração do eletrólito suporte. A ocorrência deste fenômeno pode ser atribuída às diferenças associadas com a propagação de cargas no interior da estrutura do complexo $[7,9]$. 


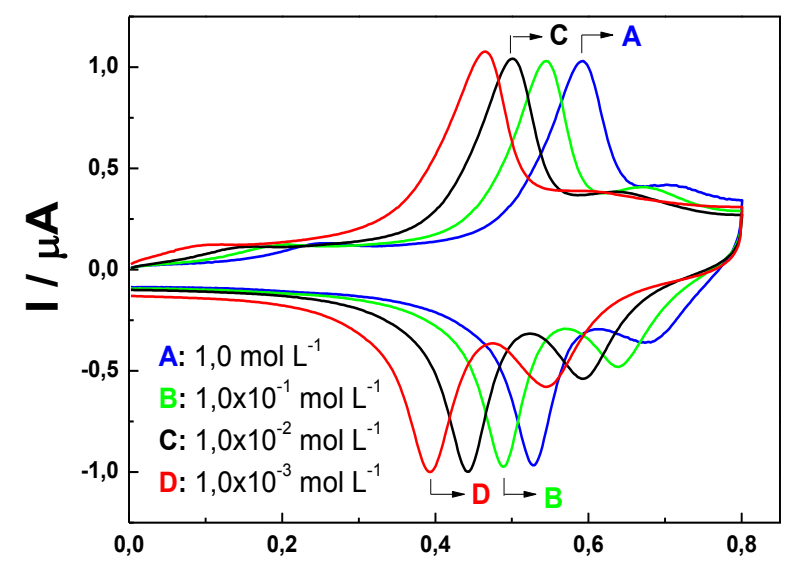

$\mathrm{E} / \mathrm{V}$ vs. Ag/AgCl

Figura 4: Perfil ciclovoltamétrico do filme de CoHCF na presença de $\mathrm{KCl}$ em diferentes concentrações: $1,0 \mathrm{~mol} \mathrm{~L}^{-1}, 1,0 \times 10^{-1} \mathrm{~mol} \mathrm{~L}^{-1}, 1,0 \times 10^{-3}$ $\mathrm{mol} \mathrm{L}^{-1}$ e $1,0 \times 10^{-3} \mathrm{~mol} \mathrm{~L}^{-1}$ numa velocidade de varredura $20 \mathrm{mV} \mathrm{s}^{-1}$.

Posteriormente, a interação entre o eletrodo modificado com CoHCF e a molécula de sulfanilamida foi realizada utilizando a técnica de voltametria cíclica, conforme mostrado na Figura 5.

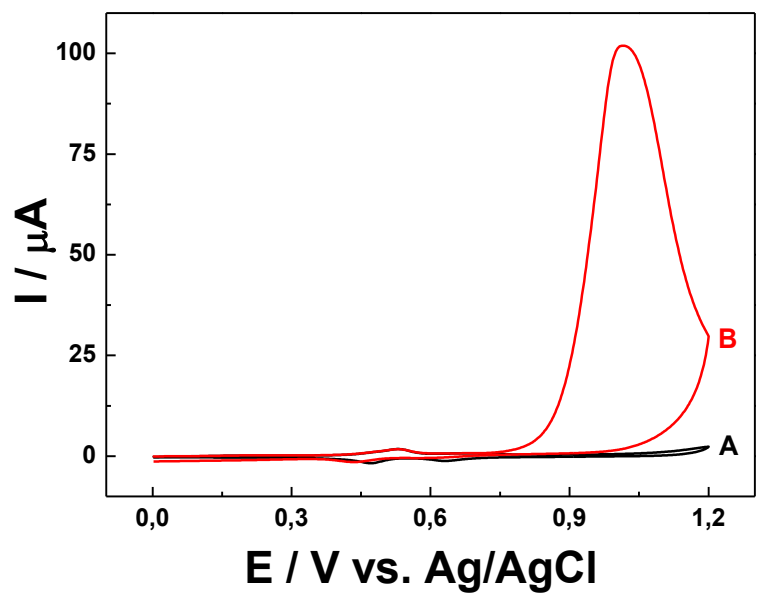

Figura 5: Perfil ciclovoltamétrico obtidos para o EQM com CoHCF na (A) ausência de sulfanilamida e na (B) presença de sulfanilamida $10 \mathrm{mmol} \mathrm{L}^{-1}$ numa velocidade de varredura de $20 \mathrm{mV} \mathrm{s}^{-1}$.

A Figura 5 apresenta o comportamento eletroquímico do eletrodo modificado com CoHCF na ausência $(A)$ e na presença $(B)$ da solução de sulfanilamida $10 \times 10^{-3}$ mol $L^{-1}$. O voltamograma cíclico $(A)$ corresponde ao eletrodo de CV modificado com CoHCF na presença de uma solução eletrolítica de $\mathrm{KCl} 0,1 \mathrm{~mol} \mathrm{~L}^{-1}$, onde 0 mesmo apresentou o comportamento característico para as condições empregadas. O voltamograma cíclico indicado pela letra (B) corresponde ao eletrodo modificado na presença da solução de sulfanilamida $10 \times 10^{-3} \mathrm{~mol} \mathrm{~L}^{-1}$, onde se observa a presença de um pico irreversível bem definido em aproximadamente $1 \mathrm{~V}$, devido a eletrooxidação da molécula de sulfanilamida (Figura 6).

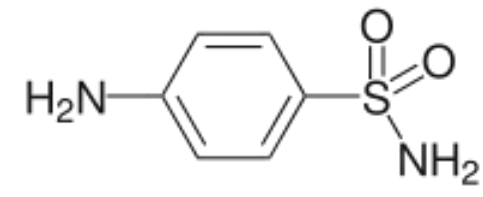

Figura 6: Estrutura da molécula de sulfanilamida.

A relação entre as concentrações de sulfanilamida e os valores de corrente obtidos foi verificada através da técnica de cronoamperometria, fixando-se o potencial em $1 \mathrm{~V}$. O resultado deste estudo é apresentado na Figura 7.

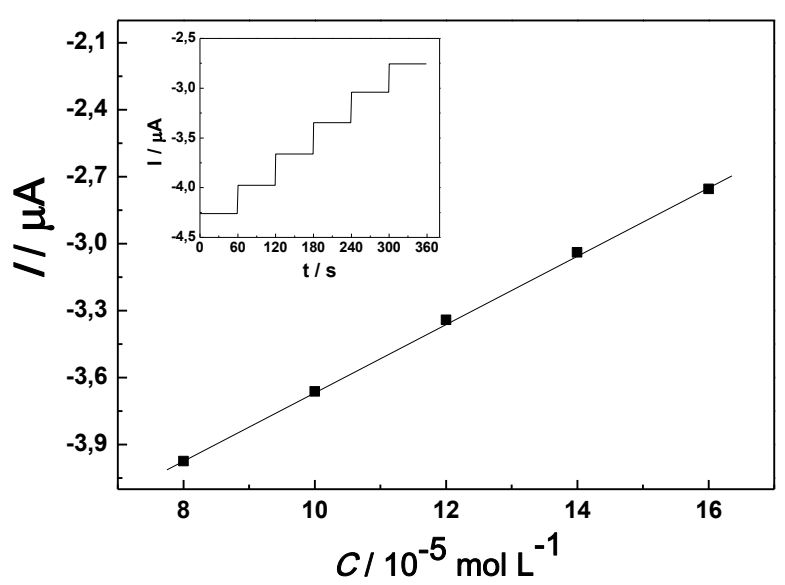

Figura 7: Relação entre as adições de sulfanilamida (nas concentrações entre 8 e $16 \times 10^{-5} \mathrm{~mol} \mathrm{~L}^{-1}$ ) e os valores das correntes (Ipa) do filme CoHCF com o potencial fixo em $1 \mathrm{~V}$.

Os valores de concentração de sulfanilamida e os valores de corrente obtidos apresentaram uma relação linear expressa pela seguinte equação: $I_{\text {pa }}(A)=0,01531 \mathrm{c}\left(\mathrm{mol} \mathrm{L}^{-1}\right)-5,19152 \times 10^{-6} \mathrm{com}$ um coeficiente de correlação $(r)$ de 0,99932 , indicando que uma resposta analítica pode ser obtida aplicando este eletrodo na detecção de sulfanilamida em diversos medicamentos.

\section{Conclusões}

Neste trabalho foi sintetizado um filme de CoHCF sobre a superfície de um eletrodo de CV e investigou-se o mesmo quanto sua estabilidade e o seu comportamento eletroquímico em diferentes eletrólitos de suporte. Além disso, verificou-se a interação desse eletrodo modificado com a molécula de sulfanilamida.

Os resultados obtidos comprovaram que o método empregado foi adequado para a modificação do eletrodo de CV com filme de CoHCF e que o mesmo demonstrou uma considerável estabilidade nas condições empregadas. Verificou-se que o comportamento eletroquímico do filme de CoHCF é fortemente influenciado pela natureza do cátion presente no eletrólito de suporte. O perfil ciclovoltamétrico do filme de CoHCF não sofreu influência significativa da natureza dos ânions, exceto pelo íon sulfato que interagiu quimicamente com o filme 
causando sua degradação, que foi comprovada pela diminuição da intensidade das correntes dos picos anódicos e catódicos.

A interação entre o eletrodo modificado e a molécula de sulfanilamida foi comprovada pela modificação do comportamento eletroquímico do filme de CoHCF, indicando a potencial aplicação deste eletrodo na detecção de sulfanilamida em diversos medicamentos.

\section{Agradecimentos}

Os autores agradecem à Fundação de Amparo à Pesquisa do Espírito Santo (FAPES) e ao Programa Institucional de Iniciação Cientifica (PIIC) da Universidade Federal do Espirito Santo.

\section{Referências}

[1] KILINC, E., GUMGUM, B., HAMAMCI, C., AYDIN F. J. Anal. Chem., V.64, p.714-720, 2009.

[2] ANVISA. Programa Nacional de Análise de Resíduos de Medicamentos Veterinários em Alimentos Expostos ao Consumo - PAMVet -. Brasilia, 2003.

[3] SOUZA, M.F.B. Quím. Nova. V.20, p.191-195, 1996.

[4] - LI, H., GAO, Q., CHEN, L., HAO, W. Sens. and Actuator B. V.173, p.540- 546, 2012.

[5] ARDUINI, F.; CASSINI, A.; AMINE, A.; RICCI, F.; MOSCONE, D.; PALLESCHI, G. J. Electroanal. Chem., V.626, p.66-74, 2009.

[6] TACCONI, N. R., RAJESHWAR, K., LEZNA, R. O. Chem. Mater. V.15, p.3046-3062, 2003.

[7] CASTILHO, M. S.; STRADIOTTO, N. R. Talanta V.74, p.1630-1634, 2008.

[8] SCHOLZ, F. DOSTAL, A. Angrew Chem Int. Ed. Engl. V.34, p.2685-2687, 1995.

[9] EFTEKHARI, A. J. Electroanal. Chem. V.537, p.59-66, 2002. 